\title{
Differences in expression of genes related to drug resistance and miRNAs regulating their expression in skin fibroblasts exposed to adalimumab and cyclosporine $A$
}

\author{
Beniamin Grabarek ${ }^{1,2,3}$, Piotr Schweizer ${ }^{1}$, Iwona Adwent ${ }^{1}$, Dominika Wcisło-Dziadecka4, Jakub Krzaczyński \\ Celina Kruszniewska-Rajs ${ }^{3}$, Joanna Gola ${ }^{3}$
}

${ }^{1}$ Katowice School of Technology, The University of Science and Art, Katowice, Poland ${ }^{2}$ Centre of Oncology, M. Sklodowska-Curie Memorial Institute, Cracow Branch, Poland ${ }^{3}$ Department of Molecular Biology, Faculty of Pharmaceutical Sciences in Sosnowiec, Medical University of Silesia in Katowice, Poland ${ }^{4}$ Department of Cosmetology, Faculty of Pharmaceutical Sciences in Sosnowiec, Medical University of Silesia in Katowice, Poland

Adv Dermatol Allergol 2021; XXXVIII (2): 249-255

DOI: https://doi.org/10.5114/ada.2019.91506

\begin{abstract}
Introduction: Adalimumab and cyclosporine A are drugs used in moderate to severe forms of psoriasis. Despite the molecular orientation of the drugs, there is a loss of adequate cell sensitivity to the anti-cytokine therapy.

Aim: To determine the changes in the gene expression profile associated with drug resistance in the culture of normal human dermal fibroblasts (NHDF) exposed to adalimumab or cyclosporine A compared to the controls. Material and methods: NHDF was exposed to adalimumab/cyclosporine A for 2, 8, $24 \mathrm{~h}$ compared to the control culture. Molecular analysis was performed using mRNA and miRNA microarray techniques. The obtained results were analysed using PL - Grid infrastructure $(p<0.05)$.

Results: Of the 22277 ID mRNA, 47 are associated with drug resistance, of which the change in expression of 17 mRNA ID is statistically significant $(p<0.05)$. The greatest change in transcriptional activity (FC $\geq 1.3)$ was observed for GLO1, SLC1OA3, TUFT1, STATH, ABCB1, AGTR1. Expression of these genes can be regulated by miR-199a-5p, miR-1231, miR-34a, miR-3188, and miR-106a (except AGTR1).

Conclusions: The analysis of changes in the expression of mRNA and miRNA related to drug resistance gives the possibility of monitoring the effectiveness of anti-cytokine therapy.
\end{abstract}

Key words: psoriasis, drug resistance, personalized therapy, miRNA.

\section{Introduction}

Psoriasis is an inflammatory, chronic and recurrent autoimmune skin disease. The onset of the disease and the severity of skin lesions mainly depend on genetic factors, and behavioural and environmental factors also play an important role [1]. The histological reflection of clinical changes is epidermal hyperplasia, parakeratosis, hypogranulosis, neutrophil and lymphocyte infiltration in the epidermis and dermis together with dilated blood vessels [2]. At the molecular level, increased secretion of proinflammatory cytokines and adipokines is observed, including interleukin (IL) IL-1, IL-2, IL-6, IL-17, IL-12 and IL-23, interferon $\gamma$ (IFN- $\gamma$ ), tumour necrosis factor $\alpha$ (TNF- $\alpha$ ), and transforming growth factor $\beta$ (TGF- $\beta$ ) [3-5].
Human dermal fibroblasts are an important model in invitro analyses that provide important new information on immunopathogenesis and the aetiology of drug resistance in personalized therapy. Fibroblasts are responsible for the production of connective tissue, moreover, they play an important role in the cell renewal system, maintaining skin integrity $[6,7]$. One of the greatest achievements of modern medicine, giving a good chance to improve the quality of life of patients with moderate to severe psoriasis, in whom conventional treatment methods used so far (phototherapy, photochemotherapy, classic anti-psoriasis drugs - methotrexate, cyclosporine $A$ (anti-IL-2 drug), acitretin, new low-molecular substances - apremilast, dimethyl fumarate) did not allow satisfactory effects to be obtained by the introduction of

Address for correspondence: Beniamin Grabarek PhD, Department of Histology, Cytophysiology and Embryology, Faculty of Medicine, University of Technology, 3-5 Park Hutniczy St, 41-800 Zabrze, Poland; Center of Oncology, M. Sklodowska-Curie Memorial Institute, Cracow Branch, 11 Garcarska St, 31-115 Krakow, Poland, phone: +48 12364 84 80, e-mail: bgrabarek7@gmail.com Received: 11.10.2019, accepted: 17.10.2019. 
biological drugs with anti-cytokine activity $[8,9]$. Among them, the recommended group of drugs is TNF- $\alpha$ inhibitors, which have become an important part of healthcare worldwide in the treatment of inflammatory diseases such as psoriasis [10]. This group includes adalimumab, which is a human monoclonal antibody that specifically binds to TNF- $\alpha$, as a result of which the signalling pathways induced by said cytokine are blocked [11, 12]. Drug resistance is a well-known phenomenon that occurs when diseases become tolerant to pharmaceutical treatment [13]. Also in the case of anti-cytokine therapy, despite the molecular focus of treatment, the phenomenon of drug resistance is observed, so it is important to analyse the network of interrelationships between cytokines contributing to the intensification of inflammation, as well as to look for new, complementary molecular markers for detection of loss of adequate response to treatment [14].

\section{Aim}

The aim of this study was to assess the effect of adalimumab on changes in the gene expression profile associated with drug resistance in Normal Human Dermal Fibroblasts (NHDF) cultures, in vitro stimulated with adalimumab at $8 \mu \mathrm{g} / \mathrm{ml}$ for $2,8,24 \mathrm{~h}$ or cyclosporine $\mathrm{A}$ at $100 \mathrm{ng} / \mathrm{ml}$ for 2, 8, $24 \mathrm{~h}$ compared to a non-drug-treated control culture. This will allow the selection of new transcripts and assessment of the possibility of their use as additional molecular markers in the diagnosis and detection of the lost response to an anti-cytokine therapy. In addition, it was assessed whether micro RNA (miRNAs) molecules could affect the expression of selected gene transcripts.

\section{Material and methods}

NHDF cell line (CC-2511; Lonza, Basel, Switzerland) was used to conduct the study. As recommended, the cells were cultured in a $5 \% \mathrm{CO}_{2}$ atmosphere at $37^{\circ} \mathrm{C}$ in a Direct Heat CO2 Incubator (Thermo Scientific, Waltham, MA, USA). FBM medium (Fibroblast Basal Medium; Lonza, Basel, Switzerland) enriched with hFGF-B (Human Fibroblast Growth Factor-basic), insulin and gentamicin (FGMTM SingleQuotsTM; Lonza, Basel, Switzerland). The experiment used NHDF cells between passage 4 and passage 6 , with a viability of $\geq 98 \%$. Cell culture was exposed to $100 \mathrm{ng} / \mathrm{ml}$ cyclosporine $A$ and $8 \mu \mathrm{g} / \mathrm{ml}$ of adalimumab for $2\left(\mathrm{H} \_2\right), 8\left(\mathrm{H} \_8\right)$, and $24\left(\mathrm{H} \_24\right) \mathrm{h}$ compared to the untreated cells (control). Selected concentrations of both drugs correspond to their average therapeutic concentration in the blood serum of patients with psoriasis.

For isolation of total RNA, TRIzol ${ }^{\circledast}$ reagent (Invitrogen Life Technologies, California, USA) was used and extractions were carried out according to the protocol recommended by the manufacturer. The extracted genetic material was subjected to quantitative (GeneQuant II spectrophotometer; Pharmacia LKB Biochrom Ltd., UK) and qualitative (agarose electrophoresis; SUBMINI K. Kucharczyk T.E., Poland) analysis.

The evaluation of the expression profile of drugrelated genes in NHDF cultures treated with drugs was performed using HG-U133A_2.0 oligonucleotide microarrays (Affymetrix, Santa Clara, CA, USA) according to the manufacturer's instructions. The following stages can be distinguished in the analysis: preparation and labelling of ribonucleic acid samples, hybridization of samples with probes present on the microarray plate and interpretation of the obtained data. The obtained results were analysed using the PL-Grid software together with the GeneSpring 12.6.1 program $(p<0.05)$. Next, in order to select miRNAs that differentiate the anti-TNF drug-exposed culture from the control culture, a miRNA microarray expression profile (GeneChip ${ }^{\circledR}$ miRNA 2.0 Arrays; Affymetrix, Santa Clara, CA) was assessed. MirTAR tool (http://mirtar.mbc.nctu.edu.tw/human/predictionlndex. php) was used to indicate which of the selected miRNAs could potentially affect the expression pattern of the analysed genes. A detailed description of the individual stages of the microarray analysis has been previously described by us [7].

\section{Results}

Based on the Affymetrix NetAffxTM Analysis Center (http://www.affymetrix.com/analysis/index.affx) after entering the query of "drug resistance" (on 10/03/2019), out of 2227 mRNA IDs present on HG- U133A_2.0 microarray, 47 mRNA IDs were related to this phenomenon. In the first stage of evaluation of the microarray profile of the analysed genes, a one-way ANOVA variance analysis test with Benjamini-Hochberg correction was performed, which gave the opportunity to compare all analysed groups of transcriptomes compared to the control culture. In addition, it became possible to indicate which of the IDs mRNAs change their expression under the influence of adalimumab or cyclosporine $A$ in a statistically significant manner $(p<0.05)$. Out of 47 mRNA IDs, in NHDF culture with adalimumab/cyclosporine A for 17 mRNA IDs observed statistically significant changes.

In the next stage, Tukey's post-hoc multiple comparison test was performed to obtain more detailed information on differences in mRNA expression in the analysed transcriptome groups. Based on the results obtained, the number of mRNA IDs differentiating the culture exposed to adalimumab compared to the control was the following H_2 vs. $\mathrm{C}=6$ mRNA IDs; H_ 8 vs. $\mathrm{C}=9 \mathrm{mRNA}$ IDs; H_24 vs. C $=1$ mRNA ID. In turn, the number of mRNA IDs differentiating the culture exposed to cyclosporine A was the following $\mathrm{H}_{-} 2$ vs. $\mathrm{C}=7$ mRNA IDs; $\mathrm{H} \_8$ vs. $\mathrm{C}=4$ mRNA IDs; H_24 vs. $\mathrm{C}=4$ mRNA IDs. 
In NHDF culture exposed to adalimumab it can be observed that 1 IDs mRNA corresponding to the DAP3 gene $(\downarrow)$ specifically differentiates NHDF culture $2 \mathrm{~h}$ after drug introduction compared to the control. In turn, in NHDF culture stimulated with cyclosporine A, it was observed that 5 mRNA IDs specifically differentiated the culture after $2 \mathrm{~h}$ : BCRA3 $(\uparrow), \operatorname{MTMR11}(\downarrow), \operatorname{TUFT1}(\downarrow), \operatorname{RRS1}(\uparrow)$, $\operatorname{SRRT}(\downarrow)$. In turn, increasing the exposure time to adalimumab to $8 \mathrm{~h}$ caused an increase in the number of mRNA IDs specifically differentiating the culture to 4 mRNA IDs that correspond to the genes: RRS1 ( $\uparrow), \operatorname{SRRT}(\uparrow)$, $\operatorname{MTMR11}(\downarrow), \operatorname{UVRAG}(\downarrow)$. In addition, increasing the exposure time of fibroblasts to cyclosporine A to 8-hours showed 3 mRNA IDs specifically differentiating the cul-

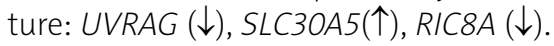

In contrast, $S S R T(\uparrow)$ is also a transcript that specifically differentiates skin fibroblasts incubated for $24 \mathrm{~h}$ with an anti-TNF drug. In turn, AGTR1 ( $\uparrow$ ) was characteristic for 24-hour exposure of cells to cyclosporine A. However, no mRNA IDs differentiating culture regardless of how long the cells were exposed to the drugs. It should be noted that the transcripts GLO1 $(\downarrow \downarrow)$, SLC1OA3 ( $\uparrow)$, TUFT1 $(\uparrow \downarrow)$, STATH $(\downarrow \downarrow), A B C B 1(\uparrow \uparrow)$ are the genes that differentiate the culture together with adalimumab ex- posed for 2 and $8 \mathrm{~h}$ while ATG14 ( $\uparrow)$ and AGTR1 ( $\uparrow$ ) are the genes that differentiate the culture together with cyclosporine A exposed for 2 and $24 \mathrm{~h}$, and TROVE2 $(\downarrow \downarrow)$ for 8 and $24 \mathrm{~h}$.

Table 1 shows changes in the direction of expression of genes differentiating culture depending on the time of exposure to adalimumab/cyclosporine A compared to non-incubated cell culture $(1.3<\mathrm{FC} \geq 1.3)$.

The last part of our work was associated with finding those micro RNA molecules (miRNAs) which might potentially influence the transcriptional activity of analysed genes (Table 2). The analysis of miRNA microarray indicated 20 miRNAs differentiating the cell culture after $2 \mathrm{~h}$ from adding the drug, compared with the control, but only for 4 miRNAs a potential impact on the expression of differentiating genes was observed. 8 hours' exposure showed that 12 miRNAs differentiated the fibroblasts culture, but for 3 miRNAs interaction with genes associated with drug resistance was confirmed. In turn, extending the incubation of cells with the drug to $24 \mathrm{~h}$ indicated 3 miRNAs differentiating the culture, but none of them had a potential effect on the expression profile of the analysed genes (Table 2).

Table 1. Changes in the transcriptional activity profile of the genes associated with drug resistance differentiating the NHDF culture exposed to adalimumab compared to the control culture $(1.3<\mathrm{FC} \geq 1.3)$

\begin{tabular}{lcccccccc}
\hline & ID & Gene symbol & $\begin{array}{c}\text { H_2 } \\
\text { (up/down) }\end{array}$ & $\begin{array}{c}\text { FC } \\
\text { (H_2 vs. C) }\end{array}$ & $\begin{array}{c}\text { H_8 } \\
\text { (up/down) }\end{array}$ & $\begin{array}{c}\text { FC } \\
\text { (H_8 vs. C) }\end{array}$ & $\begin{array}{c}\text { H_24 } \\
\text { (up/down) }\end{array}$ & $\begin{array}{c}\text { FC } \\
\text { (H_24 vs. C) }\end{array}$ \\
\hline $\begin{array}{l}\text { NHFD+ } \\
\text { adalimumab }\end{array}$ & 205807_s_at & TUFT1 & Up & +2.723 & Down & -1.117 & Up & -1.018 \\
\cline { 2 - 9 } & 209994_s_at & ABCB1 & Up & +2.081 & Up & +1.376 & Down & -1.091 \\
\cline { 2 - 9 } & 204928_s_at & SLC10A3 & Up & +1.582 & Up & +1.637 & Up & +1.162 \\
\cline { 2 - 9 } & 200681_at & GLO1 & Down & -1.278 & Down & -1.215 & Up & -1.001 \\
\hline $\begin{array}{l}\text { NHDF+ } \\
\text { cyclosporine A }\end{array}$ & 208016_s_at & STATH & Down & -1.338 & Down & -1.342 & Down & -1.037 \\
\hline
\end{tabular}

(+) overexpression of gene (increased level of MRNAs); (-) suppressed gene expression (decreased level of mRNAs); ID - ID of the probe on a microarray; FC - fold change; $\mathrm{C}$-control culture; $\mathrm{H}_{-} 2, \mathrm{H}_{-} 8, \mathrm{H}_{2} 24$ time of exposure to the drug.

Table 2. The miRNA microarray analysis of NHDF cells treated with the drug compared with a control culture

\begin{tabular}{lccccc}
\hline Compared groups & ID & Name & FC & Change & Gene \\
\hline H_2 vs. C & hsa-miR-199a-5p_st & hsa-miR-199a-5p & 3.55 & Up & $\begin{array}{c}\text { SLC1OA3 } \\
\text { GLO1 }\end{array}$ \\
\hline H_2 vs. C & hsa-miR-1231_st & hsa-miR-1231 & 2.24 & Up & GLO1 \\
\hline H_2 vs. C & hsa-miR-34a_st & hsa-miR-34a & 6.52 & Up & TUFT1 \\
\hline H_2 vs. C & hsa-miR-3188_st & hsa-miR-3188 & 2.97 & Up & TUFT1 \\
\hline H_8vs. C & hsa-miR-3188_st & hsa-miR-3188 & 2.93 & Up & TUFT1 \\
\hline H_8vs. C & hsa-miR-1231_st & hsa-miR-1231 & 2.77 & Up & GLO1 \\
\hline H_8vs. C & hsa-miR-106a_st & hsa-mir-106a & 2.45 & Up & GLO1 \\
\hline
\end{tabular}




\section{Discussion}

The premise for conducting these analyses was the observation of the loss of an adequate response to treatment during molecularly directed anti-cytokine therapy $[15,16]$. Based on the microarray technique used, it was found that a relatively small number of gene transcripts are directly related to the phenomenon of drug resistance. For example, 341 mRNA IDs present on microarray plates used in our work are bound to the TNF-induced signalling pathway [17]. This may, therefore, suggest that the process of still losing sensitivity to treatment at the molecular level is an insufficiently understood topic. This is also indicated by our observation that, although aimed at assigning the genes selected in this work to specific signalling pathways based on the PANTHER program (protein annotation through evolutionary relationship) [18], it did not indicate the association of 5 mRNA IDs that most significantly changed the profile expression, to no signal cascade. On the other hand, however, the described situation creates the opportunity to perform further, in-depth analyses in the context of molecular mechanisms of response to treatment for several key genes.

Verstockt et al., in their study on a group of 116 Crohn's disease patients treated with adalimumab, observed that some of them had drug resistance. They found this phenomenon at two critical points: 4 weeks after starting treatment and 12 weeks after the first dose. They indicate that the concentration of adalimumab in the initial period of therapy to some extent determines the subsequent potential benefits of TNF inhibitor treatment [19]. These conclusions confirm our observations carried out in vitro. The largest number of genes differentiating cultures with the drug from control was found after a short 2-hour exposure time, and then gradually decreased. In addition, also $2 \mathrm{~h}$ after adding adalimumab to NHDF cultures, the change in gene expression is the largest, and as time goes by and close to $24 \mathrm{~h}$, the level of transcriptional activity of the selected genes was similar to that observed in the control culture of skin fibroblasts.

The largest increase in transcriptional activity was observed for the TUFT1 gene coding for tuftelin, whose role has so far been discussed primarily in the context of enamel development and mineralization [20, 21]. Shilo et al. analysed the expression pattern of tuftelin at the mRNA and protein level at various stages of mouse embryo development. They observed the expression of tuftelin at the pre-tooth stage suggesting that it plays an important role in more than one cellular process. These researchers also found high dynamics of changes in the TUFT1 level during the period of their observations [22], which to some extent is also confirmed by our observations. It is also worth noting that changes in the expression profile of TUFT1 are associated with the activation of adaptive mechanisms by cells in response to hypoxia, and its potential role in the process of cell differentiation is emphasized [23]. However, considering the previously described lack of cytotoxic effect of adalimumab on NHDF culture [7], it can be assumed that the anti-TNF drug at a concentration of $8 \mu \mathrm{g} / \mathrm{ml}$, which corresponds to the average therapeutic concentration of the drug in the serum of patients treated, does not induce hypoxia in the fibroblast microenvironment. Nevertheless, it is pointed out that hypoxia is the factor that induces TUFT1 overexpression, which is an unfavourable prognostic factor. However, these observations have been made on a cancer model. They also indicate that the regulation of this gene is dependent on miR-671-5p, whose transcriptional activity is reduced in hypoxic conditions [24]. In turn, in our analysis of the miRNA microarray expression profile, we found that the expression pattern of TUFT1 is potentially regulated by 3 miRNA molecules whose level was higher in adalimumab-exposed culture compared to control. At the same time, their overexpression after $2 \mathrm{~h}$ did not affect the expression of TUFT1, which was observable after extending the time of adalimumab exposure to skin fibroblasts to $8 \mathrm{~h}$. The common miRNA molecule affecting TUFT1 transcription activity for 2 and $8 \mathrm{~h}$ incubation is miR-3188, whose level remains substantially the same while silencing expression of the regulated gene transcript. Zhou et al. emphasize that an abnormal miRNA expression pattern may be associated with the induction of treatment resistance. Based on the conducted research, they indicated that miR-3188 is involved in the development of loss of cell sensitivity to fulvestrant treatment used in oncological therapy [25]. These observations in the context of the role of miR-3188 have also recently been confirmed by Zhao et al. They observed a significant impact of miR-3188 in the development of drug resistance on chemotherapeutic treatment, linking its occurrence to the effect of this miRNA molecule on signal transduction along the mTOR/PI3K/AKT/C-JUN pathway [26]. It cannot be excluded that the concurrent increase in expression of miR-34a and miR-3188, potentially regulating TUFT1 expression after $2 \mathrm{~h}$ of incubation of cells with a drug that did not translate into a decrease in TUFT1 transcript expression, may be found in our work due to the fact that miRNA molecules should not be considered only in the context of negative expression regulators, but also its enhancement. This seems justified as in some therapeutic strategies it is aimed to reduce the level of a given miRNA through anti-miRNA molecules, binding in terms of complementarity with miRNA [27].

Research conducted by Bobek et al. indicate that the role of tuftelin in particular biological and cellular processes depends on the type of cells [28]. This is confirmed by the observations of Shipeng et al. in an invitro fibroblast model where they observed an increase in TUFT1 expression ( $F C=2.4$ ) in response to their exposure to low intensity red light [29]. Also in our analysis we found overexpression of TUFT1 $2 \mathrm{~h}$ after the introduction 
of the anti-TNF drug into culture, which, in conjunction with previous reports [7, 24, 28, 29] suggests that in fibroblasts under the influence of an external factor, the level of TUFT1 will increase. Interesting, in the context of our work are the observations of Natsumi et al., who emphasize the possibility of using changes in expression of TUFT1 not only as a complementary molecular marker, but also as a new, attractive therapeutic target. They indicate that the expression pattern of the discussed gene and the encoded protein correlated with the response to anticancer treatment both in vitro and in vivo [30]. This observation indicates the usefulness of cell culture analyses and the possibility of referring in vitro results to in vivo analyses.

Also, the $A B C B 1$ gene can be doubled in expression after introduction of adalimumab into NHDF culture. The mechanism of action and the emergence of drug resistance with the participation of proteins encoded by this gene is the removal of xenobiotics with significant specificity for the substrate from the cell (the protein is an ATP dependent pump), as a result of which a decrease in drug concentration in the body is observed [31]. Observations of Boyer et al. indicate that higher $A B C B 1$ expression contributes to the induction of drug resistance. At the same time, they emphasize that the gene in question should not be directly linked to loss of response to treatment as silencing its expression did not significantly improve the results of pharmacotherapy [32]. Thus, $A B C B 1$ overexpression may indicate a group of patients in whom drug resistance should be expected. In this way, alternative therapy paths could be developed at the beginning of the implementation of a given therapeutic strategy, which emphasizes the need to develop and implement personalization of treatment, in which molecular analyses are extremely important [33]. The last of the genes for which an increase in transcriptional activity was observed was $S \angle C 10 A 3$, which plays an important role in the transport of the drug into the cell [34]. Kirschmeyer et al. observed, like us, that the expression profile of the gene in question can be regulated by miRNAs. They found that miR$146 b-5 p$ reduced the number of mRNA SLC1OA3 copies [35], which was not confirmed by our research. Under the influence of the addition of adalimumab to culture, we have observed that hsa-miR-199a-5p potentially affects the expression of SLC1OA3. However, it must also be noted that this miRNA molecule interacts with two gene transcripts: SLC1OA3 and GLO1. Perhaps, therefore, despite the potentially possible interaction of SLC1OA3: miR-199a-5p, the complementarity of the resulting complex is small. Foulkes et al. suggest that the expression pattern of SLC1OA3 is dependent on TNF- $\alpha$ concentration observing a decrease in its activity with an increase in cytokine concentration [36]. Therefore, reducing TNF- $\alpha$ concentration by its neutralization with adalimumab resulted in the overexpression of SLC1OA3, which was confirmed by the results obtained in this paper.
Analysis of the microarray profile of mRNA and miRNA for the selected genes showed that the GLO1 gene can be regulated by three miRNA molecules: miR-1231, miR106a, miR-199-5p. Regardless of time, we found a reduction in its expression in response to the addition of adalimumab to NHDF cultures. Glyoxalase 1 (GLO1; S-dlactoylglutathione lyase, EC 4.4.1.5) is a part of the glyoxalase system, which, in addition to GLO1, consists of GLO2 (hydroxyacyl glutathione hydrolase, EC 3.1.2.6). This enzyme is responsible for the transformation of cytotoxic oxo-aldehyde, i.e. methylglyoxal (MGO) is d-lactate [37]. Hutschenreuther et al. indicate that overexpression of GLO1 is characteristic of cancer cells in which excessive amounts of MGO accumulate. They also link the increase in expression of this gene with the induction of hypoxia in the tumour microenvironment [38]. Therefore, our silencing of GLO1 expression under the influence of adalimumab suggests that this drug does not cause adverse changes in cell metabolism and its microenvironment, which in consequence could cause cells to acquire cancer. However, in the previous paper, we observed an increase in expression of the survivin-encoding gene, a protein that basically does not exist under physiological conditions [17]. This confirms the occurrence of complex changes and interrelationships between genes, and also constitutes a premise for further analyses, all the more that the analysis of changes in the GLO1 expression pattern is associated with predicting response to treatment $[39,40]$. We have observed that miR-1231 may affect GLO1 expression after 2 and $8 \mathrm{~h}$ of exposure of dermal fibroblasts to adalimumab. The increase in miR-1231 expression is another premise for the safety of adalimumab and the relatively low risk of induction of carcinogenesis [41, 42].

In contrast, in the fibroblasts culture exposed to cyclosporine A, our results showed that the only gene associated with the phenomenon of resistance that meets the assumptions $1.3<\mathrm{FC} \geq 1.3$ is the gene AGTR1 encoding Angiotensin II receptor sub-type 1 . It is interesting that the highest differences in the expression profile of this gene was observed after 24 hours' incubation of NHDF culture with the drug, while under adalimumab treatment, the effect was observed earlier. Yamani et al. described that overexpression of AGTR1 was associated with response to rejection after transplantation [43]. Observations by $\mathrm{Ma}$ et al. showed that AGTR1 can be a useful supplementary marker and explained that overexpression of this gene was strongly associated with promoting epithelial-mesenchymal transition [44], what also showed that during cyclosporine therapy a higher risk of carcinogenesis might be observed [45]. These and our results suggest that during the cyclosporine A treatment, a drug resistance phenomenon may be observed, however its frequency is lower compared with the adalimumab therapy.

Nevertheless, it should be noted that these assumptions apply to skin fibroblasts, which were the research 
model in our work. Therefore, in further analysis, we intend to determine changes in the expression of selected genes and miRNAs in the clinical material.

\section{Conclusions}

Adalimumab and cyclosporine A affect the transcriptional activity of genes associated with drug resistance. However, our analysis shows that the phenomenon of drug resistance is more pronounced with adalimumab than cyclosporine A. A thorough understanding and observation of the molecular mechanisms associated with the cellular response to adalimumab will help us better understand the complex nature of autoimmune diseases.

\section{Acknowledgments}

This research was financed by the Medical University of Silesia in Katowice/Poland on the basis of decision no. KNW-1-032/N/9/I.

\section{Conflict of interest}

The authors declare no conflict of interest.

\section{References}

1. Szepietowski J, Adamski Z, Chodorowska G, et al. Leczenie łuszczycy - rekomendacje ekspertów Polskiego Towarzystwa Dermatologicznego. Część II: łuszczyca umiarkowana do ciężkiej. Dermatol Rev 2014; 101: 455-72.

2. Menter A, Gottlieb SR, Feldman AS, et al. Bhushan Guidelines of care for the management of psoriasis and psoriatic arthritis: Section 1. Overview of psoriasis and guidelines of care for the treatment of psoriasis with biologics. J Am Acad Dermatol 2008; 58: 826-50.

3. Monin L, Gaffen SL. Interleukin 17 family cytokines: signaling mechanisms biological activities and therapeutic implications. SH Perspect Biol 2018; 10: a028522.

4. Arruda L, Ypiranga S, Martins GA. Systemic treatment of psoriasis - Part II: Biologic immunomodulator agents. An Bras Dermatol 2004; 79: 393-408.

5. Wcisło-Dziadecka D, Kaźmierczak A, Grabarek B, et al. Are new variants of psoriasis therapy (IL-17 inhibitors) safe? Int J Dermatol 2019; 58: 1360-5.

6. Patlolla A, Knighten BS, Tchounwou P. Multi-walled carbon nanotubes induce cytotoxicity genotoxicity and apoptosis in normal human dermal fibroblast cells. Ethn Dis 2010; 20 (1 Suppl 1): 65-72.

7. Wcisło-Dziadecka D, Grabarek B, Zmarzły N, et al. Influence of adalimumab on the expression profile of genes associated with the histaminergic system in the skin fibroblasts in vitro. BioMed Res Int 2018; 2018: 1582173.

8. Reich A, Szepietowski J, Adamski Z, et al. Łuszczyca. Rekomendacje diagnostyczno-terapeutyczne Polskiego Towarzystwa Dermatologicznego. Część II: łuszczyca umiarkowana do ciężkiej. Dermatol Rev 2018; 105: 329-57.

9. Reich A, Adamski Z, Chodorowska G, et al. A. Łuszczyca. Rekomendacje diagnostyczno-terapeutyczne Polskiego Towarzystwa Dermatologicznego. Część I: łuszczyca łagodna. Dermatol Rev 2018; 105: 225-43.
10. Pouw MF, Krieckaert CL, Nurmohamed MT. Key findings towards optimising adalimumab treatment: the concentration - effect curve. Ann Rheum Dis 2015; 74: 513-8.

11. Bartelds GM, Wijbrandts CA, Nurmohamed MT, et al. Clinical response to adalimumab: relationship to anti-adalimumab antibodies and serum adalimumab concentrations in rheumatoid arthritis. Ann Rheum Dis 2007; 66: 921-5.

12. Mitoma H, Horiuchi T, Tsukamoto H, Ueda N. Molecular mechanisms of action of anti-TNF-alpha agents - comparison among therapeutic TNF-alpha antagonists. Cytokine 2018; 101: 56-63.

13. Housman G, Byler S, Heerboth S, et al. Drug resistance in cancer: an overview. Cancers 2014; 6: 1769-92.

14. Wcisło-Dziadecka D, Grabarek B, Brzezińska-Wcisło L, Mazurek $U$. Drug resistance in anti-TNF therapy of psoriatic arthritis. Dermatol Rev 2018; 105: 625-31.

15. Wcisło-Dziadecka D, Zbiciak M, Wcisło-Brzezińska L, Mazurek U. Anti-cytokine therapy for psoriasis - not only TNF blockers Overview of reports on the effectiveness of therapy with IL12/IL23 and T and B lymphocyte inhibitors. Post Hig Med Dosw 2016; 70: 1198-205.

16. Powrozik B, Kubowicz P, Pękala E. Przeciwciała monoklonalne w terapii celowanej. Post Hig Med Dosw 2012; 66: 663-73.

17. Wcisło-Dziadecka D, Gola J, Grabarek B, et al. Effect of adalimumab on the expression of genes encoding TNF-alpha signal paths in skin fibroblasts in vitro. Adv Dermatol Allergol 2018; 35: 413-22.

18. Mi H, Muruganujan A, Casagrande JT, Thomas PD. Large-scale gene function analysis with the PANTHER classification system. Nature Protocols 2013; 8: 1551-66.

19. Verstockt B, Moors G, Bian S, et al. Ferrante Influence of early adalimumab serum levels on immunogenicity and long-term outcome of anti-TNF naive Crohn's disease patients: the usefulness of rapid testing. Aliment Pharmacol Ther 2018; 48: 731-9.

20.Deutsch D, Leiser Y, Shay B, et al. The human tuftelin gene and the expression of tuftelin in mineralizing and nonmineralizing tissues. Connect Tissue Res 2002; 43: 425-34.

21. Küchler EC, Dea Bruzamolin C, Ayumi M, et al. Polymorphisms in nonamelogenin enamel matrix genes are associated with dental fluorosis. Caries Res 2018; 52: 1-6.

22. Shilo D, Blumenfeld A, Haze A, et al. Tuftelin's involvement in embryonic development. J Exp Zool B Mol Dev Evol 2019; 332: 125-35.

23. Leiser $Y$, Silverstein N, Blumenfeld A, et al. The induction of tuftelin expression in PC12 cell line during hypoxia and NGFinduced differentiation. J Cell Physiol 2011; 226: 165-72.

24. Changwei D, Zhenyu Z, Qiuran X, et al. Hypoxia-induced TUFT1 promotes the growth and metastasis of hepatocellular carcinoma by activating the Ca2+/PI3K/AKT pathway. Oncogene 2019; 38: 1239-55.

25.Zhou Q, Zeng H, Ye P, et al. Long Differential microRNA profiles between fulvestrant-resistant and tamoxifen-resistant human breast cancer cells. Anti-cancer Drug 2018; 29: 539 48.

26. Mengyang Z, Rongcheng L, Yiyi L, et al. miR-3188 regulates nasopharyngeal carcinoma proliferation and chemosensitivity through a FOXO1-modulated positive feedback loop with mTOR-p-PI3K/AKT-c-JUN. Nat Commun 2016; 7: 11309.

27. Mie Y, Hirano Y, Kowata K, et al. Function control of antimicroRNA oligonucleotides using interstrand cross-linked duplexes. Mol Ther Nucleic Acids 2018; 10: 64-74. 
28. Bobek J, Oralova V, Kratochvilova A, et al. Tuftelin and HIFs expression in osteogenesis. Histochem Cell Biol 2019; 152: 355-63.

29. Shipeng S, Yaou Z, Chi-Chun F, et al. cDNA microarray analysis of gene expression profiles in human fibroblast cells irradiated with red light. J Invest Dermatol 2003; 120: 849-57.

30. Natsumi K, Kazunobu I, Shingo D, et al. TUFT1 interacts with RABGAP1 and regulates mTORC1 signaling. Cell Discov 2018; 4: 1 .

31. Ambudkar SV, Kim IW, Sauna ZE. The power of the pump: mechanisms of action of P-glycoprotein ABCB1. Eur J Pharm Sci 2006; 27: 392-400.

32. Boyer T, Gonzales F, Barthélémy A, et al. Clinical significance of ABCB1 in acute myeloid leukemia: a comprehensive study. Cancers 2019; 11: 1323.

33. Tavakolpour S, Darvishi M, Ghasemiadl M. Pharmacogenetics: a strategy for personalized medicine for autoimmune diseases. Clin Genet 2018; 93: 481-97.

34. Spratlin J, Sangha R, Glubrecht D, et al. The absence of human equilibrative nucleoside transporter 1 is associated with reduced survival in patients with gemcitabine-treated pancreas adenocarcinoma. Clin Cancer Res 2004; 10: 6956-61.

35. Kirchmeyer M, Servais FA, Hamdorf M, et al. Cytokine-mediated modulation of the hepatic miRNome: miR-146b-5p is an IL-6-inducible miRNA with multiple targets. J Leukoc Biol 2018; 104: 987-1002.

36. Foulkes MJ, Henry KM, Rougeot J, et al. Expression and regulation of drug transporters in vertebrate neutrophils. Sci Rep 2017; 7: 4967.

37. Thornalley PJ, Waris S, Fleming T, et al. Imidazopurinones are markers of physiological genomic damage linked to DNA instability and glyoxalase 1-associated tumour multidrug resistance. Nucleic Acids Res 2010; 38: 5432-42.

38. Hutschenreuther A, Bigl M, Hemdan N, et al. Modulation of GLO1 expression affects malignant properties of cells. Int J Mol Sci 2016; 17: 2133.

39. Ranganathan S, Walsh ES, Tew KD. Glyoxalase I in detoxification: studies using a glyoxalase I transfectant cell line. Biochem J 1995; 309: 127-31.

40. Davies GF, Roesler WJ, Juurlink BH, Harkness TA. Troglitazone overcomes doxorubicin-resistance in resistant k562 leukemia cells. Leuk Lymphoma 2005; 46: 1199-206.

41. Zhang J, Zhang J, Qiu W, et al. MicroRNA-1231 exerts a tumor suppressor role through regulating the EGFR/PI3K/AKT axis in glioma. Neuro Oncol 2018; 139: 547-62.

42. Wang H, Wu J, Luo WJ, Hu JL. Low expression of miR-1231 in patients with glioma and its prognostic significance. Nat Neurosci 2018; 22: 8399-405.

43. Yamani MH, Cook DJ, Rodriguez ER, et al. Increased expression of angiotensin II type 1 receptor AGTR1 in heart transplant recipients with recurrent rejection. J Heart Lung Transplant 2006; 2511: 1283-9.

44. Ma Y, Xia Z, Ye C, et al. AGTR1 promotes lymph node metastasis in breast cancer by upregulating CXCR4/SDF-1alpha and inducing cell migration and invasion. Aging 2019; 1112: 3969-92.

45. Dando EE, Anvekar RA. Psoriasis therapies and the risk of cutaneous malignancy. J Cut Med 2017; 12: 55-63. 